\title{
Guillain Barre and Anesthesia- Intraoperative Disaster?
}

\author{
Omonele O. Nwokolo*, Semhar J. Ghebremichael, Sara Guzman-Reyes \\ Department of Anesthesiology, University of Texas-Houston Medical School, Houston, USA \\ Email: *Omonele.O.Nwokolo@uth.tmc.edu, Semhar.Gebremichael@uth.tmc.edu, \\ Sara.Guzman-Reyes@uth.tmc.edu
}

Received 2 August 2015; accepted 20 August 2015; published 26 August 2015

Copyright (C) 2015 by authors and OALib.

This work is licensed under the Creative Commons Attribution International License (CC BY). http://creativecommons.org/licenses/by/4.0/

CC) $\underset{\mathrm{EY}}{\mathrm{C}}$ Open Access

\section{Abstract}

This is the case of a $76 \mathrm{y} / \mathrm{o}$ AA man with a past medical history of Hypertension and Guillain Barre Syndrome (GBS), who presented for a median sternotomy and excision of a thymic mass after workup for multiple episodes of syncope revealed the thymic mass. Intraoperatively, the patient had 2 episodes of cardiac arrest with asystole. He was adequately resuscitated and extubated. He was later determined by Neurology to have severe autonomic dysfunction and not a candidate for any elective surgery.

\section{Keywords}

Guillain Barre, Autonomic Dysfunction, Median Sternotomy: Paravertebral Block

Subject Areas: Anaesthesiology \& Pain Management

\section{Case Presentation}

This is a 76-year-old African American male with past medical history of HTN and GBS presenting for median sternotomy and excision of a thymic mass. The patient had some weakness from GBS and was currently ambulating via a walker. He had 3 prior episodes of syncope, which prompted an Emergency Room visit for evaluation. There was no explanation for the syncopal episodes found on workup, however, a $2.7 \mathrm{~cm}$ by $3.6 \mathrm{~cm}$ thymic mass was noted. He underwent radiation therapy for the thymoma. The mass shrunk to $1 \mathrm{~cm}$ by $2 \mathrm{~cm}$ on subsequent chest CT. Despite this, patient continued to have deterioration in muscle strength thus Thoracic Surgery was consulted for removal of the mass with the assumption of myasthenia gravis.

Other preoperative workup included an unremarkable holter study, normal Echo (EF of 60\% - 64\% with no valvular abnormalities) and, no significant stenotic lesions on doppler ultrasound of bilateral carotids. CT and MRI brain showed minor degenerative changes with no signs of stroke or bleed.

"Corresponding author. 
The case was to proceed after discussion with the CT surgeon, acknowledging that there was no cardiopulmonary bypass capability in the hospital.

On the day of surgery, patient presented appears younger than his stated age. He reports being stronger now and has progressed from wheelchair to ambulation with a walker. Patient is also able to lie flat without shortness of breath. Patient stated that he was extremely nervous about the surgery and concerned he may not "wake up" from surgery. The anesthesia and surgical team had an in-depth discussion regarding the risks and benefits as well as the overall plan for anesthesia and post op management. This alleviated the patient's anxiety.

Bilateral paravertebral catheters were placed in the OR uneventfully. A test dose of $1.5 \%$ Lidocaine with 1:200,000 Epinephrine 2 cc each was done with no signs of intravascular or intrathecal catheter placement. A left radial arterial line was placed, and the patient was induced with Lidocaine $100 \mathrm{mg}$, Fentanyl $100 \mathrm{mcg}$ and Ketamine $50 \mathrm{mg}$ total in $25 \mathrm{mg}$ increments maintaining spontaneous respiration. An 8.0 endotracheal tube was placed via a flexible fiber optic scope. No masses or collapses noted during intubation. After securing the ETT, the surgeon performed a bronchoscopy with no significant findings. Vital signs remained stable and the patient was positioned and prepped for a right-sided internal jugular vein central venous catheter. During the prep, the blood pressure began to decrease precipitously with no significant change in heart rate. The prep was stopped; phenylephrine $200 \mathrm{mcg}$ was given to the patient with no response. The blood pressure continued to decline and $\mathrm{EtCO}_{2}$ was noted to have decreased as well. EKG showed asystole. Anesthesia STAT was called overhead. ACLS was started with chest compressions and Epinephrine 1mg IV was given. The patient responded quickly, the blood pressure improved to 140s systolic. A quick differential diagnosis was performed for cause of event. Bilateral breath sounds were present. Another bronchoscopy was performed and it was unremarkable. Arterial blood gases with chemistries sent were all within normal limits.

We proceeded to place the central line with no further events and the decision was made with the surgical team to monitor him for a short time prior to proceeding with the surgery. Approximately 10 minutes after central line placement, on 0.4 MAC of sevoflurane, the patient repeated the episode, becoming progressively hypotensive, and then asystole. It again required chest compression and Epinephrine $1 \mathrm{mg}$ IV with return of spontaneous circulation. The decision was made to abort the procedure, and attempt to emerge the patient from anesthesia. The patient emerged, with adequate tidal volumes and appropriate vital signs. A trial of extubation was done in which he immediately opened his eyes and asked if he could go home. A neurologic exam was performed and patient was intact with no new deficits. The paravertebral catheters were discontinued; he was transferred to the ICU.

A full workup was performed in the ICU; cardiac enzymes where negative, chemistries and hematologic labs were normal. EKG and echocardiogram were unchanged from preoperative exams. The neurology team came back to evaluate the patient, and on review of the intraoperative events, diagnosed the patient with severe autonomic dysfunction due to GBS and should not undergo any elective surgical procedures.

\section{Discussion}

Guillain-Barre Syndrome is a rare disorder presenting as an acute demyelinating polyneuropathy. This typically occurs as an autoimmune response after a respiratory or gastrointestinal infection [1]. Respiratory failure can occur in these patients with up to $30 \%$ requiring invasive mechanical ventilation and up to $10 \%$ having long term neurologic sequelea [2]. Its clinical manifestations can include severe autonomic dysfunction including orthostatic hypotension, uncontrolled hypertension and sudden death [3]. This combination of manifestations make this subset of patients very challenging for the anesthesiologist in the perioperative setting. Preoperatively, this patient exhibited signs suggested autonomic dysfunction. Perhaps the syncopal episodes could have been explained by severe hypotension or arrhythmias that resolved prior to EKG evaluations. The addition of a thymic mass in this patient added a layer of intrigue.

The risks of this procedure were discussed fully with the Cardiothoracic Surgeon. At our institution there is no capability of cardiopulmonary bypass if needed. The surgical team agreed that the mass had decreased in size therefore did not to pose a significant operative risk to the patient.

The anesthetic management of these patients can be challenging. The first goal was preparing the patient for extubation. The patient had a history of weakness and pneumonia. The aim was to tailor an anesthetic plan that would limit the time the patient spent on the ventilator. We wanted adequate pain control to decrease splinting and aid in extubation; however, there have been several reports in the literature of exacerbation of symptoms of Guillain-Barre Syndrome after neuroaxial blocks [4]-[6]. Therefore, bilateral paravertebral catheters were placed 
for post op pain control.

Despite the decreased size of the mediastinal mass, we felt it would be safer to induce this patient and maintain spontaneous respiration when securing the airway due to the possibility of mechanical obstruction when airway and neck muscles are relaxed [7]-[9]. We elected not to perform an awake fiberoptic intubation because of signs of a good airway and the patient's ability to lie flat without any airway obstruction. Induction agents were titrated to maintain spontaneous respiration and the airway secured via flexible fiber optic scope. This also allowed the surgeons to perform a bronchoscopy. We also decided not to use paralytic agents due to his weakness and the unpredictability of his response to them [12].

ACLS was appropriately followed when the patient decompensated and went into asystole. There was a differential diagnosis workup to elicit the cause of the event. We considered bilateral pneumothoraxes after placement of the paravertebral catheters. However, patient had bilateral and equal breath sounds. A bronchoscopy did not reveal any compression in the airway. The initial decision to continue the case was due to patient's quick response to treatment. However after a second episode with no discernible cause, we felt it prudent to abort the case. The decision to have a trial of extubation was predicated on the fact that the patient met extubation criteria after the anesthetic agents dissipated and the initial plan to decrease the amount of mechanical ventilation time for him.

\section{Conclusion}

The autonomic dysfunction from Guillain-Barre syndrome can be profound. This could have explained the multiple episodes of syncope that the patient had prior to the discovery of the thymic mass. This can also explain both intraoperative events; patient becoming severely hypotensive progressing to asystole. The lesson learned is to be highly selective of Guillain-Barre patients as surgical candidates for elective surgery as autonomic dysfunction can cause significant morbidity and mortality intraoperatively. This patient is currently stable and managed medically by neurology.

\section{References}

[1] Fletcher, D.D., Lawn, N.D., Wolter, T.D. and Wijdicks, E.F. (2000) Long-Term Outcome in Patients with GuillainBarre Syndrome Requiring Mechanical Ventilation. Neurology, 54, 2311-2315. http://dx.doi.org/10.1212/WNL.54.12.2311

[2] Schottlender, J.G., Lombardi, D., Toledo, A., Otero, C., Mazia, C. and Menga, G. (1999) Respiratory Failure in GuillainBarre Syndrome. Medicina, 59, 705-709.

[3] Lichtenfeld, P. (1971) Autonomic Dysfunction in the Guillain-Bad Syndrome. The American Journal of Medicine, 50, 772-780. http://dx.doi.org/10.1016/0002-9343(71)90185-9

[4] Chan, L.Y.S., Tsui, M.H.Y. and Leung, T.N. (2004) Guillain-Barré Syndrome in Pregnancy. Acta Obstetricia et Gynecologica Scandinavica, 83, 319-325.

[5] Kocabas, S., Karaman, S., Firat, V., et al. (2007) Anesthetic Management of Guillain-Barré Syndrome in Pregnancy. Journal of Clinical Anesthesia, 19, 299-302. http://dx.doi.org/10.1016/j.jclinane.2006.09.008

[6] Köller, H., Kieser, B.C., Jander, S., et al. (2005) Chronic Inflammatory Demyelinating Polyneuropathy. The New England Journal of Medicine, 352, 1143-1156. http://dx.doi.org/10.1056/NEJMra041347

[7] Hines, R.L. and Marschall, K. (2008) Stoelting's Anesthesia and Co-Existing Disease, 5th Edition, Elsevier Saunders, Philadelphia, Chapter 9.

[8] Miller, R.D., Eriksson, L.I., Fleisher, L., Wiener-Kronish, J.P. and Young, W.L. (2009) Miller's Anesthesia. 7th Edition, Churchill Livingstone, London, Chapter 59.

[9] Weiss, S.J. and Ochroch, E.A. (2009) Longnecker's Anesthesiology. McGraw Hill Medical, New York, Chapter 53.

[10] Pandey, C.K., Bose, N., Garg, G., Singh, N., Baronia, A., Agarwal, A., Singh, P.K. and Singh, U. (2002) Gabapentin for the Treatment of Pain in GuillainBarre Syndrome: A Double-Blinded, Placebocontrolled, Crossover Study. Anesthesia \& Analgesia, 95, 1719-1723. http://dx.doi.org/10.1097/00000539-200212000-00046

[11] Morgan, G.E., Mikhail, M.S. and Murray, M.J. (2008) Neuromuscular Blocking Agents, Clinical Anesthesiology. 4th Edition, McGraw Hill, USA, 178-197.

[12] Hara, K., Minami, K., Takamoto, K., Shiraishi, M. and Sata, T. (2000) The Prolonged Effect of a Muscle Relaxant in a Patient with Chronic Inflammatory Demyelinating Polyradiculoneuropathy. Anesthesia \& Analgesia, 90, 224-226. http://dx.doi.org/10.1097/00000539-200001000-00045 